\title{
Structure of Channel Quantization Codebook for Multiuser Spatial Multiplexing Systems
}

\author{
Behrouz Khoshnevis and Wei Yu \\ Department of Electrical and Computer Engineering \\ University of Toronto, Toronto, Ontario, Canada \\ Email: \{bkhoshnevis, weiyu\}@comm.utoronto.ca
}

\begin{abstract}
This paper studies the structure of the channel quantization codebook for multiuser MISO systems with limited channel state information at the base-station. The problem is cast in the form of minimizing the sum power subject to the worst-case SINR constraints over spherical channel uncertainty regions. This paper adopts a zero-forcing approach for beamforming vectors design, and uses a robust optimization technique via semidefinite programming (SDP) for power control as the benchmark performance measure. We then present an alternative less complex and practically feasible method for computing the power values and present sufficient conditions on the uncertainty radius so that the resulting sum power remains close to the SDP solution. The proposed conditions guarantee that the interference caused by the channel uncertainties can be effectively controlled. Based on these conditions, we study the structure of the channel quantization codebooks and show that the quantization codebook has a product form that involves spatially uniform quantization of the channel direction, and independent channel magnitude quantization which is uniform in $\mathrm{dB}$ scale. The structural insight obtained by our analysis also gives a bit-sharing law for dividing the quantization bits between the two codebooks. We finally show that the total number of quantization bits should increase as $\log \left(\mathbf{S I N R}_{\text {target }}\right)$ as the target SINR increases.
\end{abstract}

\section{INTRODUCTION}

A base-station with multiple antennas can potentially serve multiple users simultaneously, a capability usually referred to as spatial multiplexing. To perform this effectively, however, the base-station requires certain degrees of channel state information (CSI) in order to safely distinguish users spatially and perform power control accordingly. CSI is typically obtained at the remote terminals and fedback to the base-station via a rate-limited feedback link. The fact that exact CSI may not be available in practice motivates the study of the downlink spatial multiplexing systems with imperfect CSI at the base station.

For fixed-rate delay-constrained types of communication, a reasonable problem formulation is to minimize the total transmission power subject to signal-to-interference-plus-noise ratio (SINR) constraints at the users' side. Assuming perfect CSI at the base-station, this problem is extensively studied in the literature and several algorithms are proposed for finding the optimum powers and beamforming vectors [1], [2].

The counterpart of this problem for the imperfect CSI case can be cast in the form of a robust optimization problem that minimizes the sum power subject to the worst-case SINR constraints over the channel uncertainty regions. The uncertainty regions are usually assumed to be ellipsoidal regions around the nominal user channels. This problem and similar robust precoding problems have attracted a lot of attention lately and numerous algorithms and reformulations are proposed. The authors of [3] fix the beamforming vectors and show that the robust minimization of power, subject to the users' mean-squared-error (MSE) constraints, can be transformed to a convex problem and hence the powers can be computed efficiently. In [4], the authors provide a rank-relaxation method to transform the robust SINR-constrained problem to a convex semidefinite programming (SDP) problem. Similarly, the authors of [5] propose different levels of relaxation leading to SDP problems with different levels of complexity. The same authors also present robust formulations for MSE-constrained problem [6]. Finally, the authors of [7] propose an ellipsoid method for solving the robust SINR-constrained problem.

Although the robust design problems studied in these papers can usually be transformed to or approximated by a convex problem and therefore result in computationally feasible beamforming and power control algorithms, these approaches are not necessarily easy to implement in practice and more importantly they do not provide insight to the structure of the problem solution. Structural insights are important because one of the key questions in the design of spatial multiplexing systems for advanced wireless standards is the problem of channel quantization codebook design. The lack of structural insights limits the applicability of the aforementioned robust optimization studies to this area.

This paper aims to make progress in the design of channel quantization codebooks. We focus on multiuser multiple-input single-output (MISO) systems, and base our approach on a simple suboptimal solution to the beamforming and power control problem resulting from the bounding of the SINR terms. The beamforming vectors are assumed to be zeroforcing directions for the quantized directions and the power levels are determined in a robust manner such that the target SINR constraints are satisfied for the worst-case interference scenarios. This approach provides us with a sufficient condition on the radius of uncertainty balls such that the multiuser interference can be effectively controlled. Based on this condition, we derive the structure of the channel quantization codebook for effective interference management. We show that

- the channel quantization codebook should have a product structure, consisting of a channel direction quantizer 
which is uniform spatially and a channel magnitude quantizer which is uniform in $\mathrm{dB}$ scale;

- the number of quantization bits devoted to spatial direction quantization should be $(M-1)$ times the number of bits devoted to magnitude quantization, where $M$ is the number of base-station antennas;

- the total number of the quantization bits (per user) should scale as logarithm of the target SINR.

\section{Beamforming and Power Control with Perfect CSI: OUTAGE IS INEVITABLE}

Consider a downlink MISO channel with $M$ antennas at the base station (BS) and $M$ users each with a single antenna. Let $\mathbf{h}_{i} \in \mathbb{R}^{M}$ denote the user channels for $1 \leq i \leq M$. Let $P_{i}, \mathbf{v}_{i}$, and $\gamma_{i}$ denote the power, the unit beamforming vector, and the SINR constraint for user $i$. The minimization of the total transmit power subject to the user SINR constraints is formulated as follows:

$$
\begin{aligned}
& \min _{P_{i}, \mathbf{v}_{i}} \sum_{i=1}^{M} P_{i} \\
& \text { s.t. } \frac{P_{i}\left|\mathbf{h}_{i}^{T} \mathbf{v}_{i}\right|^{2}}{\sum_{k \neq i} P_{k}\left|\mathbf{h}_{i}^{T} \mathbf{v}_{k}\right|^{2}+1} \geq \gamma_{i}, \quad i=1,2, \cdots, M
\end{aligned}
$$

where the noise power at all users is assumed to be 1 . Note that the computations in this paper are done in the real space for simplicity. Extension to the complex space is straightforward.

It is well known that this problem can be transformed to a convex second order cone programming (SOCP) problem [2]. It can also be solved via an iterative power-beamforming update algorithm based on the uplink-downlink duality [1]. A simple alternative to these optimal solutions is to use the zeroforcing (ZF) beamforming vectors to remove the interference and compute the power levels to satisfy the SINR constraint with equality. We will adopt the ZF suboptimal solution throughput this paper.

An important matter to consider with this formulation is that if the user channels are independent with uniformly distributed directions and the BS is required to satisfy the SINR constraints for all channel realizations, it is not difficult to see that the average transmission power will be unbounded. This happens because when the channels for different users are in near alignment with each other, it is difficult to separate them spatially.

To see this rigorously, define $\theta_{i}=\angle\left(\mathbf{h}_{i}, \mathbf{H}_{-i}\right)$, where

$$
\mathbf{H}_{-i}=\operatorname{span}\left(\left\{\mathbf{h}_{k} \mid k \neq i\right\}\right) .
$$

Assume that the users' channel directions $\mathbf{h}_{i} / \mid \mathbf{h}_{i} \|$ are spatially uniform and are independent of the channel magnitudes $\left\|\mathbf{h}_{i}\right\|$. Then $\theta_{i}$ is uniformly distributed in $\left[0, \frac{\pi}{2}\right]$ and is independent of $\left\|\mathbf{h}_{i}\right\|$. The average sum power of ZF method is therefore given by

$$
P_{\text {ave }}=\mathbb{E}\left\{P_{\text {ZF,sum }}\right\}=\sum_{i=1}^{M} \gamma_{i} \mathbb{E}\left\{1 /\left\|\mathbf{h}_{i}\right\|^{2}\right\} \mathbb{E}\left\{1 / \sin ^{2}\left(\theta_{i}\right)\right\} .
$$

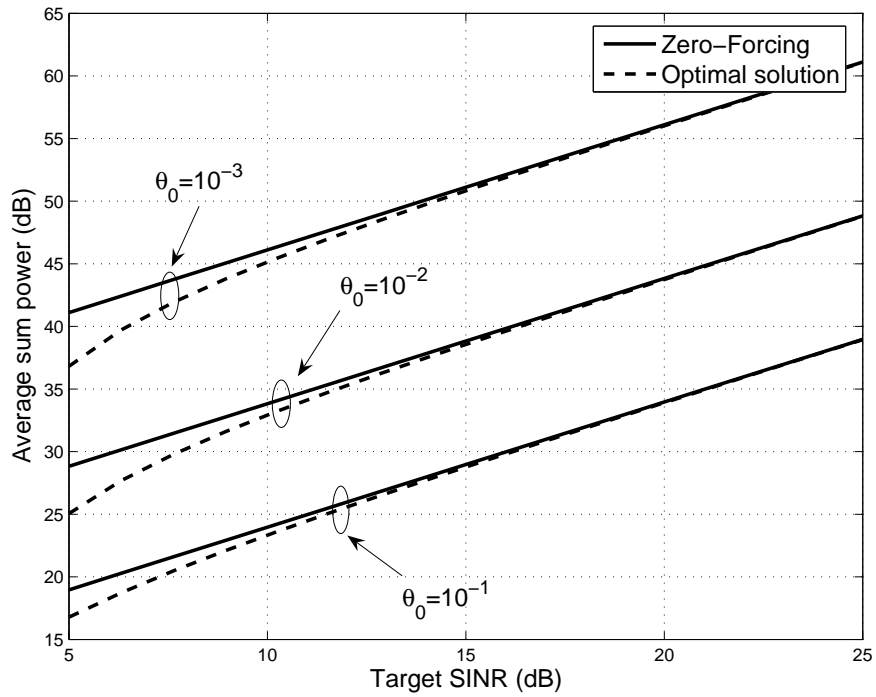

Fig. 1. Sum power vs. the target SINR. Sum power is averaged over $10^{4}$ channel realizations; $M=3$; the user channels are i.i.d. $\mathbf{h}_{i} \sim \mathcal{N}\left(0, \mathbf{I}_{M}\right)$.

As $\theta_{i}$ is uniformly distributed in $\left[0, \frac{\pi}{2}\right]$, the expectation of $1 / \sin ^{2}\left(\theta_{i}\right)$ becomes unbounded.

To avoid this, we have to accept a certain degree of outage. A reasonable approach is to declare outage when

$$
0 \leq \theta_{i} \leq \theta_{0},
$$

where $\theta_{0}$ is the smallest acceptable angle between $\mathbf{h}_{i}$ and $\mathbf{H}_{-i}$ for all $i$. Throughout this paper, we will refer to this condition as the $\theta_{0}$-condition. If this condition is violated for some $i$, the BS declares an outage.

Noting that $\theta_{i}$ is uniform on $\left[0, \frac{\pi}{2}\right]$, it is easy to verify that the average sum power and the system outage probability values are as follows:

$$
\begin{aligned}
& P_{\text {ave }}=\frac{2 \cot \left(\theta_{0}\right)}{\pi} \sum_{i=1}^{M} \gamma_{i} \mathbb{E}\left\{1 /\left\|\mathbf{h}_{i}\right\|^{2}\right\} \approx \frac{2 M}{\pi} \frac{\bar{P}}{\theta_{0}} \\
& p_{\text {out }} \leq \frac{2 M}{\pi} \theta_{0}
\end{aligned}
$$

where $\bar{P}=\frac{1}{M} \sum_{i=1}^{M} \gamma_{i} \mathbb{E}\left\{1 /\left\|\mathbf{h}_{i}\right\|^{2}\right\}$ and the approximation holds for small $\theta_{0}$.

By fixing the probability of system outage or equivalently $\theta_{0}$, one can perform a fair comparison of the ZF solution and the optimal solution of [1], [2]. As shown in Fig. 1, the ZF solution approaches the optimal solution at high-SINR regime.

\section{BeAmforming AND Power Control With CSI UNCERTAINTY}

In practice, the CSI available to $\mathrm{BS}$ is associated with some degree of uncertainty. This can result from channel quantization in frequency-division-duplex (FDD) systems or from estimation errors and outdated estimations in the reverse channel training in time-division-duplex (TDD) systems. In order to maintain the quality of the communication in spite of the uncertainty, the BS should find the beamforming vectors 
and power levels by solving the following robust optimization problem:

$$
\begin{aligned}
& \min _{P_{i}, \mathbf{v}_{i}} \sum_{i=1}^{M} P_{i} \\
& \text { s.t. } \inf _{\mathbf{h}_{i} \in S_{i}} \frac{P_{i}\left|\mathbf{h}_{i}^{T} \mathbf{v}_{i}\right|^{2}}{\sum_{k \neq i} P_{k}\left|\mathbf{h}_{i}^{T} \mathbf{v}_{k}\right|^{2}+1} \geq \gamma_{i}, \quad i=1,2, \cdots, M
\end{aligned}
$$

where $S_{i}$ is the uncertainty region associated with $i$ th user's channel. In this paper we assume spherical regions:

$$
S_{i}=\left\{\mathbf{h} \mid\left\|\mathbf{h}-\hat{\mathbf{h}}_{i}\right\| \leq r_{i}\right\}
$$

where $\hat{\mathbf{h}}_{i}$ is the nominal user channel and $r_{i}$ is the uncertainty radius.

An analytic solution to this problem is not yet available. However, if we fix the beamforming vectors $\mathbf{v}_{i}$ (e.g. via a zero-forcing approach, where $\mathbf{v}_{i}$ 's are the normalized columns of $\hat{\mathbf{H}}^{-T}$ and $\hat{\mathbf{H}}=\left[\hat{\mathbf{h}}_{1}|\cdots| \hat{\mathbf{h}}_{M}\right]$ ) finding the power values $P_{i}$ can be shown to reduce to the following SDP problem:

$$
\begin{aligned}
\min _{P_{i}, \lambda_{i}} & \sum_{i=1}^{M} P_{i} \\
\text { s.t. } & \lambda_{i}\left[\begin{array}{cc}
\mathbf{I}_{M} & -\hat{\mathbf{h}}_{i} \\
-\hat{\mathbf{h}}_{i}^{T} & \left\|\hat{\mathbf{h}}_{i}\right\|^{2}-r_{i}^{2}
\end{array}\right] \\
& -\left[\begin{array}{cc}
\sum_{k \neq i} P_{k} \mathbf{v}_{k} \mathbf{v}_{k}^{T}-\frac{1}{\gamma_{i}} \mathbf{v}_{i} \mathbf{v}_{i}^{T} & 0 \\
0 & 1
\end{array}\right] \succeq 0, \\
& P_{i} \geq 0, \quad \lambda_{i} \geq 0, \quad i=1,2, \cdots, M .
\end{aligned}
$$

Problem (4) is in fact a special case of the SDP problem derived in [4] for ellipsoid uncertainty regions. The equivalence of the problem (3) and (4) is proven by using the $S$-procedure [8].

Although the SDP problem can be numerically solved in an efficient manner, it is not necessarily feasible in practice. Moreover, it does not provide any insight into the structure of the problem solution and therefore cannot be directly used to derive the channel quantization codebooks. To overcome this, we resort to less complex methods which act as upper and lower bounds for the SDP solution.

\section{A. Upper Bound Solution}

To achieve an upper bound on the total transmission power, we bound the SINR terms as follows:

$$
\begin{aligned}
& \inf _{\mathbf{h}_{i} \in S_{i} \frac{P_{i}\left|\mathbf{h}_{i}^{T} \mathbf{v}_{i}\right|^{2}}{\sum_{k \neq i} P_{k}\left|\mathbf{h}_{i}^{T} \mathbf{v}_{k}\right|^{2}+1}} \geq \frac{\inf _{\mathbf{h}_{i} \in S_{i}} P_{i}\left|\mathbf{h}_{i}^{T} \mathbf{v}_{i}\right|^{2}}{\sup _{\mathbf{h}_{i} \in S_{i}} \sum_{k \neq i} P_{k}\left|\mathbf{h}_{i}^{T} \mathbf{v}_{k}\right|^{2}+1} \\
& \geq \frac{\inf _{\mathbf{h}_{i} \in S_{i}} P_{i}\left|\mathbf{h}_{i}^{T} \mathbf{v}_{i}\right|^{2}}{\sum_{k \neq i} P_{k} \sup _{\mathbf{h}_{i} \in S_{i}}\left|\mathbf{h}_{i}^{T} \mathbf{v}_{k}\right|^{2}+1} .
\end{aligned}
$$

By putting the last term equal to the target SINR $\gamma_{i}$, and solving for $P_{i}$ values, one can achieve an upper bound on the sum power. To simplify (5), we use the following lemma.
Lemma 1: Let $S=\{\mathbf{h} \mid\|\mathbf{h}-\hat{\mathbf{h}}\| \leq r\}$ and $\mathbf{v}$ be an arbitrary vector. Then

$$
\begin{aligned}
& \sup _{\mathbf{h} \in S}\left|\mathbf{h}^{T} \mathbf{v}\right|=\left|\hat{\mathbf{h}}^{T} \mathbf{v}\right|+r \\
& \inf _{\mathbf{h} \in S}\left|\mathbf{h}^{T} \mathbf{v}\right|=\max \left(\left|\hat{\mathbf{h}}^{T} \mathbf{v}\right|-r, 0\right) .
\end{aligned}
$$

Let $y_{i}=\left\|\hat{\mathbf{h}}_{i}\right\|$ and $\theta_{i}=\angle\left(\hat{\mathbf{h}}_{i}, \hat{\mathbf{H}}_{-i}\right)$, where

$$
\hat{\mathbf{H}}_{-i}=\operatorname{span}\left(\left\{\hat{\mathbf{h}}_{k} \mid k \neq i\right\}\right) \text {. }
$$

Also let $\mathbf{v}_{i}$ be the zero-forcing directions for the nominal channel directions $\hat{\mathbf{h}}_{i}$. Then $\left|\hat{\mathbf{h}}_{i}^{T} \mathbf{v}_{i}\right|=y_{i} \sin \theta_{i}$, which for now we assume to be larger than $r_{i}$. We also have $\left|\hat{\mathbf{h}}_{i}^{T} \mathbf{v}_{k}\right|=0$ for $k \neq i$, since $\mathbf{v}_{i}$ 's are the ZF vectors for $\hat{\mathbf{h}}_{i}$ 's. By combining these with Lemma 1 and putting (5) equal to $\gamma_{i}$, we get to the following set of linear equations for $P_{i}$ 's:

$$
\frac{P_{i}\left(y_{i} \sin \theta_{i}-r_{i}\right)^{2}}{\left(\sum_{k \neq i} P_{k}\right) r_{i}^{2}+1}=\gamma_{i},
$$

After a few computations, the following upper bound on the sum power is achieved:

$$
P_{\mathrm{ub}, \mathrm{sum}}=\frac{\sum_{i} \alpha_{i}}{1-\sum_{i} r_{i}^{2} \alpha_{i}},
$$

where $\alpha_{i}=\frac{\gamma_{i}}{\left(y_{i} \sin \theta_{i}-r_{i}\right)^{2}+\gamma_{i} r_{i}^{2}}$.

\section{B. Lower Bound Solution}

We can bound the SINR term by ignoring the interference:

$$
\inf _{\mathbf{h}_{i} \in S_{i}} \frac{P_{i}\left|\mathbf{h}_{i}^{T} \mathbf{v}_{i}\right|^{2}}{\sum_{k \neq i} P_{k}\left|\mathbf{h}_{i}^{T} \mathbf{v}_{k}\right|^{2}+1} \leq \inf _{\mathbf{h}_{i} \in S_{i}} P_{i}\left|\mathbf{h}_{i}^{T} \mathbf{v}_{i}\right|^{2} .
$$

By putting this bound equal to the target SINR $\gamma_{i}$, we achieve the following lower bound for the sum power:

$$
P_{\mathrm{lb}, \mathrm{sum}}=\sum_{i} \frac{\gamma_{i}}{\left(y_{i} \sin \theta_{i}-r_{i}\right)^{2}} .
$$

We clearly have $P_{\mathrm{lb} \text {,sum }}<P_{\mathrm{SDP} \text {,sum }}<P_{\mathrm{ub} \text {,sum. }}$. The following theorem guarantees the closeness of these sum-power values if the uncertainty radii are small compared to the channel magnitudes.

Theorem 1: Let $c<\frac{M}{M-1}$. If

$$
r_{i} \leq \frac{\sin \theta_{i}}{1+\sqrt{\frac{M}{c} \gamma_{i}}} y_{i}
$$

then

$$
\frac{P_{\mathrm{ub}, \mathrm{sum}}}{P_{\mathrm{lb}, \mathrm{sum}}} \leq \frac{1}{1-\frac{c}{1+c / M}} \approx 1+c,
$$

where the approximation holds for small $c$. Since $P_{\mathrm{lb} \text {,sum }}<$ $P_{\mathrm{SDP}, \text { sum }}<P_{\mathrm{ub}, \text { sum }}$, the same bound works for $\frac{P_{\mathrm{ub}, \text { sum }}}{P_{\mathrm{SD}, \text { sum }}}$ and $\frac{P_{\mathrm{SDP}, \text { sum }}}{P_{\mathrm{lb} \text { sum }}}$.

Proof: From (9), we have $\gamma_{i} r_{i}^{2} \leq \frac{c}{M}\left(y_{i} \sin \theta_{i}-r_{i}\right)^{2}$ and

$$
\frac{\gamma_{i} r_{i}^{2}}{\left(y_{i} \sin \theta_{i}-r_{i}\right)^{2}+\gamma_{i} r_{i}^{2}} \leq \frac{c / M}{1+c / M} .
$$




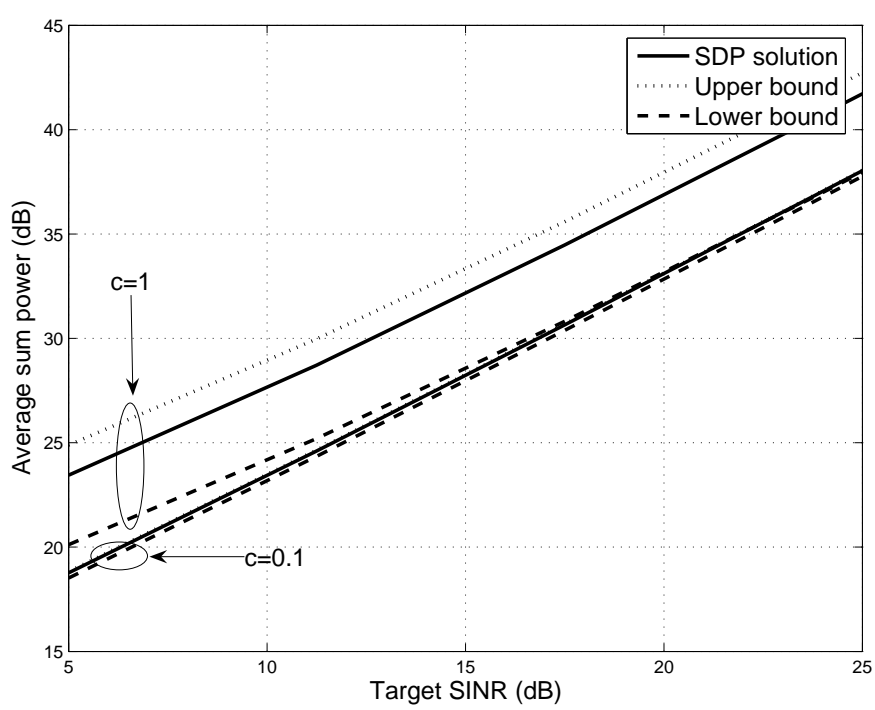

Fig. 2. Sum power vs. the target SINR for SDP, lower bound, and upper bound power solutions. Sum powers are averaged over 100 channel realizations; $\theta_{0}=0.1 ; M=3$; the user channels are i.i.d. $\mathbf{h}_{i} \sim \mathcal{N}\left(0, \mathbf{I}_{M}\right)$.

Therefore,

$$
\begin{aligned}
P_{\mathrm{ub}, \mathrm{sum}}=\frac{\sum_{i} \frac{\gamma_{i}}{\left(y_{i} \sin \theta_{i}-r_{i}\right)^{2}+\gamma_{i} r_{i}^{2}}}{1-\sum_{i} \frac{\gamma_{i} r_{i}^{2}}{\left(y_{i} \sin \theta_{i}-r_{i}\right)^{2}+\gamma_{i} r_{i}^{2}}} & \leq \frac{\sum_{i} \frac{\gamma_{i}}{\left(y_{i} \sin \theta_{i}-r_{i}\right)^{2}}}{1-M \frac{c / M}{1+c / M}} \\
& =\frac{P_{\mathrm{lb}, \mathrm{sum}}}{1-\frac{c}{1+c / M}} .
\end{aligned}
$$

According to Theorem 1, if the uncertainty radii are small, the upper bound and lower bound solutions will be close to the optimal SDP solution. Fig. 2 compares the performance of the three solutions, when condition (9) is satisfied. The figure shows that the upper bound power solutions can be used as low-complexity approximations to the optimal SDP solutions.

On the other hand, the closeness of the SDP solution to the lower bound solution means that the interference can be safely ignored. Therefore, the condition (9) provides a sufficient condition for successful interference removal by zero-forcing, even in the presence of channel uncertainty. This condition is utilized in the next section to derive the structure of the appropriate channel quantization codebooks for operating close to the no-interference regime.

\section{Structure of the Channel Quantization CODEBOOK}

In this section, we study the structure of the channel quantization codebook. We assume that the quantization (uncertainty) regions are approximately spherical and that the channel direction is uniformly distributed over the unit sphere in $\mathbb{R}^{M}$.

The criterion used for codebook design is qualitative rather than quantitative. We are looking for a codebook structure that guarantees a performance close to the no-interference regime. For this purpose, we adopt the condition (9) of Theorem 1. For the cases that the system in not in outage, we have $\theta_{i}>\theta_{0}$ according to the $\theta_{0}$-condition. Since user $i$ is not aware of $\theta_{i}$, the codebook structure should be designed for the worst interference case $\theta_{i}=\theta_{0}$. We therefore use the following rule as the scaling law between the uncertainty radius (quantization region radius) and the channel magnitude:

$$
r=\beta y,
$$

where $\beta=\frac{\sin \theta_{0}}{1+\sqrt{\frac{M}{c} \gamma}}$. Recall that $y=\|\hat{\mathbf{h}}\|$, and $r$ is the radius of the uncertainty ball. Also note that $\beta \ll 1$ if $\theta_{0} \ll 1$. The user index $i$ is dropped in this section for simplicity.

A key observation based on (11) is that the radius of the uncertainty ball should scale linearly with the magnitude of the channel quantization codeword. This suggests that the channel quantization codebook should have a product structure as shown in Fig. 3, where the channel magnitude and the channel direction can be quantized independently.

The channel magnitude quantizer can be designed as follows. First, we choose the quantization range based on the outage probability, i.e. choose real numbers $b>a>0$ such that

$$
\operatorname{Prob}\left\{\|\mathbf{h}\| \in[a, b]^{c}\right\} \ll p_{\text {out }},
$$

where the outage probability is governed by the value of $\theta_{0}$ as described in Section II, with $\mathbf{h}_{i}$ 's replaced by $\hat{\mathbf{h}}_{i}$ 's.

Now, let $y^{(n)}$ denote the center of the quantization spheres for $1 \leq n \leq N_{p}$, where $N_{p}$ is the number of quantization levels in the direction of the channel magnitude. From Fig. 3, we have

$$
y^{(n+1)}-y^{(n)}=r^{(n)}+r^{(n+1)} .
$$

By applying (11), $y^{(n)}$ 's form a geometric sequence:

$$
y^{(n)}=\frac{a}{1+\beta}\left(\frac{1+\beta}{1-\beta}\right)^{n} .
$$

This means that the magnitude quantization levels are uniformly spaced in $\mathrm{dB}$ scale.

The number of quantization bits for magnitude quantization is given by:

$$
\begin{aligned}
B_{p}=\log _{2} N_{p} & =\log _{2}\left(\frac{\ln (b / a)}{\ln ((1+\beta) /(1-\beta))}\right) \\
& \approx \log _{2}(\ln (b / a))+\log _{2} \frac{1}{\beta} \approx \log _{2} \frac{1}{\beta},
\end{aligned}
$$

where the approximations hold for small $\beta$ (and reasonable values of $a$ and $b$ ).

For the channel direction quantizer, the codebook structure of Fig. 3 suggests that the direction quantization vectors should be independent of the channel magnitude quantizer. The number of direction quantization points $N_{b}$ can be computed via a sphere packing argument:

$$
\begin{aligned}
N_{b} & =\frac{\frac{1}{2}\left(\left(y^{(n)}+r^{(n)}\right)^{M}-\left(y^{(n)}-r^{(n)}\right)^{M}\right)}{\left(r^{(n)}\right)^{M}} \\
& =\frac{1}{2 \beta^{M}}\left((1+\beta)^{M}-(1-\beta)^{M}\right) \approx \frac{M}{\beta^{M-1}},
\end{aligned}
$$




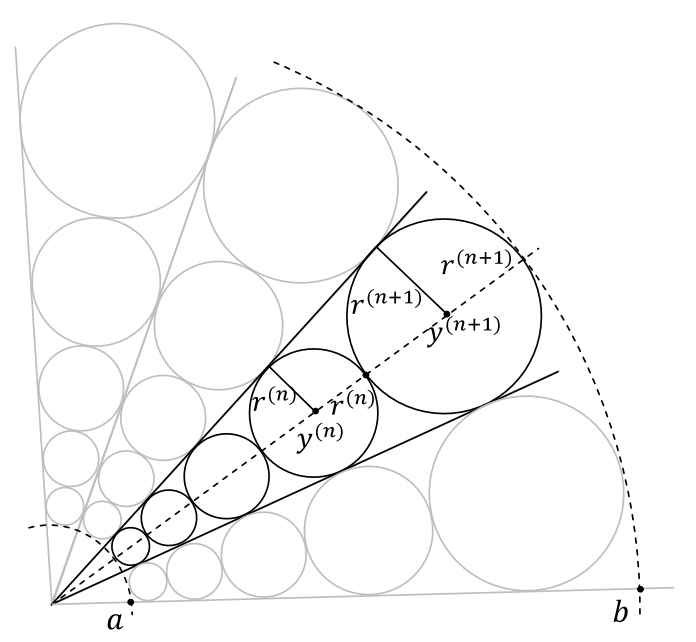

Fig. 3. Approximate channel quantization codebook structure with spherical quantization regions.

where we use the fact that the volume of a sphere in $M$ dimensional space is proportional to its radius to the $M$ th power. The approximation in the last step above holds for small $\beta$. The $\frac{1}{2}$ factor comes from the fact we only need to quantize half the space, i.e. $\mathbf{h}$ and $\mathbf{- h}$ are equivalent for our purposes. Therefore for $\beta \ll 1$ :

$$
B_{b}=\log _{2}\left(N_{b}\right) \approx(M-1) \log _{2} \frac{1}{\beta} .
$$

By combining (12) and (13) we achieve the following bitsharing rule between the channel magnitude and direction quantization codebooks:

$$
B_{b} \approx(M-1) B_{p} .
$$

Although this result directly relies on the assumption that the quantization regions are spherical, it reflects a fundamental principle in the multi-user communications: in order to effectively mitigate the interference and operate close to the no-interference regime, every dimension of the $(M-1)$ dimensional channel direction space should be quantized almost as finely as the channel magnitude. This is in direct contrast to the single-user case. For the single-user system, in the asymptotic regime where SNR tends to infinity, more bits should be used for magnitude quantization, since the mismatch between the channel direction and the quantized directions can be compensated with a bounded power [9].

To summarize, the channel quantization codebook should be expressed as the product of a uniform direction quantization codebook and a uniform (in $\mathrm{dB}$ scale) magnitude quantization codebook. The number of bits devoted to channel direction quantization should be $(M-1)$ times the number of bits devoted to channel magnitude quantization, where $M$ is the number of base-station antennas.

We end this section by deriving the dependence of the total number of quantization bits on the target SINR. Achieving a higher target SINR requires further mitigation of interference and consequently more precise CSI. From the definition of $\beta$, we have $\beta \approx \frac{\kappa}{\sqrt{\gamma}}$, where $\kappa=\sqrt{\frac{c}{M}} \sin \theta_{0}$. Therefore,

$$
\begin{aligned}
B=B_{b}+B_{p} \approx M \log _{2} \frac{1}{\beta} \approx M \log _{2} \frac{1}{\kappa}+\frac{M}{2} \log _{2} \gamma \\
\\
\approx \frac{M}{2} \log _{2} \gamma,
\end{aligned}
$$

as $\gamma \rightarrow \infty$. Thus the total number of quantization bits should scale linearly with the target SINR (in dB scale). A similar result is reported in [10], although for a different problem setup. The scaling rule (15) also dictates how the feedback link bandwidth should be divided among the users with different target SINR constraints in a FDD system.

\section{CONCLusions}

This paper studies the robust power control and beamforming problem for the multiuser downlink MISO system with channel uncertainties at the BS. We assume a zeroforcing beamforming design and propose a simple and practical method for power control with channel uncertainty. Further, we give a sufficient condition that guarantees effective mitigation of multiuser interference in spite of channel uncertainty. We use this condition to study the structure of the channel quantization codebook for operating near the nointerference regime. The codebook structure turns out to be the product of a uniform direction quantizer and a uniform (in $\mathrm{dB}$ scale) channel magnitude quantizer. We also present a bit-sharing law between the two codebooks. Finally, we show that the total number of the quantization bits should scale as $\log \left(\mathrm{SINR}_{\text {target }}\right)$ as the target SINR increases.

\section{REFERENCES}

[1] F. Rashid-Farrokhi, K. R. Liu, and L. Tassiulas, "Transit beamforming and power control for cellular wireless systems," IEEE J. Select. Areas Commun., vol. 16, pp. 1437-1450, Oct. 1998.

[2] A. Wiesel, Y. C. Eldar, and S. Shamai, "Linear precoding via conic optimization for fixed MIMO receivers," IEEE Trans. Signal Process., vol. 54, pp. 161-176, Jan. 2006.

[3] M. Payaró, A. Pascual-Iserte, and M. A. Lagunas, "Robust power allocation designs for multiuser and multiantenna downlink communication systems through convex optimization," IEEE J. Sel. Areas Commun., vol. 25, pp. 1390-1401, Sep. 2007.

[4] G. Zheng, K. K. Wong, and T. S. Ng, "Robust linear MIMO in the downlink: a worst-case optimization with ellipsoidal uncertainty regions,' Eurasip Journal on Advances in Signal Processing, vol. 2008, pp. 1-15, 2008.

[5] M. Botros Shenouda and T. N. Davidson, "Convex conic formulations of robust downlink precoder designs with quality of service constraints," IEEE J. Select. Topics Signal Processing, vol. 1, pp. 714-724, Dec. 2007.

[6] M. Botros Shenouda, T. N. Davidson, "On the design of linear transceivers for multiuser systems with channel uncertainty," IEEE J. Select. Areas Commun., vol. 26, pp. 1015-1024, Aug. 2008.

[7] N. Vucic and H. Boche, "Downlink precoding for multiuser MISO systems with imperfect channel knowledge," in Proc. IEEE Int. Conf. Acoust., Speech and Sig. Proc., Mar.-Apr. 2008, pp. 3121-3124.

[8] S. Boyd and L. Vandenberghe, Convex Optimization. Cambridge University Press, 2004.

[9] B. Khoshnevis and W. Yu, "Joint power control and beamforming codebook design for MISO channels with limited feedback," IEEE Globecom 2009, Nov.-Dec. 2009.

[10] N. Jindal, "MIMO broadcast channels with finite-rate feedback," IEEE Trans. Inform. Theory, vol. 52, pp. 5045-5060, Nov. 2006. 